\title{
COMPUTATIONAL ANALYSIS OF A RAREFIED HYPERSONIC FLOW OVER COMBINED GAP/STEP GEOMETRIES
}

\author{
P. H. M. Leite and W. F. N. Santos \\ Combustion and Propulsion Laboratory (LCP) \\ National Institute for Space Research (INPE) \\ Cachoeira Paulista-SP 12630-000, Brazil
}

\begin{abstract}
This work describes a computational analysis of a hypersonic flow over a combined gap/step configuration at zero degree angle of attack, in chemical equilibrium and thermal nonequilibrium. Effects on the flowfield structure due to changes on the step frontal-face height have been investigated by employing the Direct Simulation Monte Carlo (DSMC) method. The work focuses the attention of designers of hypersonic configurations on the fundamental parameter of surface discontinuity, which can have an important impact on even initial designs. The results highlight the sensitivity of the primary flowfield properties, velocity, density, pressure, and temperature due to changes on the step frontal-face height. The analysis showed that the upstream disturbance in the gap/step configuration increased with increasing the frontal-face height. In addition, it was observed that the separation region for the gap/step configuration increased with increasing the step frontal-face height. It was found that density and pressure for the gap/step configuration dramatically increased inside the gap as compared to those observed for the gap configuration, i. e., a gap without a step.
\end{abstract}

\section{INTRODUCTION}

The presence of surface discontinuities, such as protuberances, cavities, gaps, or steps, in modern aerodynamic configurations occurs as a desired or undesired design feature. These discontinuities, due to the interaction with external flows, may contribute to physical phenomena related to the problem of flow separation. When separation occurs in high Mach number flows, changes on the pressure distribution acting on the surface and on the heat transfer rate to the surface can have catastrophic effects on the vehicle. The presence of hot spots at separation and reattachment points changes the characteristics of the flow over the vehicle

This is an Open Access article distributed under the terms of the Creative Commons Attribution License 4.0, which permits unrestricted use, distribution, and reproduction in any medium, provided the original work is properly cited. 
and can cause, for instance, failure in the thermal protection system of the vehicle.

Hypersonic vehicles are thermally protected during the reentry in the Earth atmosphere by a thermal protection system made by a large number of tiles on their structures. Among these individual tiles, cavities or gaps may arise. In addition, it is possible to occur a misalignment among tiles. This tile misalignment may configure an undesired discontinuity on the vehicle surface.

The extensive literature on surface discontinuities [1-14] is devoted primarily to experimental and computational studies dealing with flows on protuberances, cavity, gap, forward- and backward-facing steps. For the purpose of this introduction, it will be sufficient to describe only a few of these studies.

Bertran and Wiggs [1] investigated experimentally the effect of distortions, consisting of small protuberances and holes, on the wing of a hypersonic vehicle. The effect on the pressure and on the heat flux to the surface was investigated for a range of Mach number from 7 to 10 and angle of attack up to $20^{\circ}$. Results showed that these surface distortions presented a lower influence on the pressure rather than on the heat-flux distributions. They also showed that all distortions investigated caused an increase in the aerodynamic heating.

Rogers and Berry [2] conducted an experimental investigation on forwardfacing steps in a supersonic flow, $\mathrm{M}_{\infty} \approx 2$, characterized by a thick laminar boundary layer. Free-stream pressure was defined at 30, 50, and $70 \mu \mathrm{m} \mathrm{Hg}$, covering a Reynolds number per inch between 98 and 281. Eight-step heights were tested, ranging in 0.1 -inch intervals from 0.1 to $0.9 \mathrm{in}$, except $0.8 \mathrm{in}$. In addition, the step height was comparable with the local boundary-layer thickness for a flat-plate without steps. According to them, the investigation showed that the largest pressure rise occurred at the step face. Furthermore, it was found that this pressure rise depends on height-to-length $(h / L)$ ratio, where $h$ is the step height and $L$ is the distance from the flat-plate leading edge to the step.

Bertin and Keisner [6] investigated experimentally the effect of a step and a gap tile misalignment on transition locations in the plane of symmetry of the Space Shuttle Orbiter. Data were obtained for a Mach number of 8 and angle of attack of $30^{\circ}$. Data correlations relating the location of the boundary-layer transition on the vehicle surface were presented in the continuum flow regime. They concluded that the step height is more effective in the boundary-layer transition than the gap length.

Hinderks et al. [14] have investigated the gap flow structure. They showed that a complex flow exists within the gap, consisting of a vortex superposed by an axial flow. The analysis showed that the heat flux transferred to the structure depends on the thermal state of the structure. Also, the effects due to changes in the gap geometry caused by deformations in the gap structure demonstrated that deformations should be considered in the design analysis.

In general, these research studies have been conducted in order to understand, among others, the physical aspects of a laminar or turbulent boundary layer in 
a subsonic, supersonic, or hypersonic flow past to these types of discontinuities characterized by a sudden change on the surface slope. The major interest in these research studies has gone into considering laminar or turbulent flow in the continuum flow regime. However, there is little understanding of the physical aspects of rarefied hypersonic flows past to these discontinuities related to the severe aerothermodynamic environment associated with a reentry vehicle.

In attempting to assess the effect of these discontinuities in the transition flow regime, Paolicchi and Santos [15] have studied a rarefied hypersonic flow over a flat plate with a gap by employing the DSMC method. The work was motivated by the interest in investigating the gap length-to-depth $(L / H)$ ratio effects on the flowfield structure and on the aerodynamic surface quantities. The primary emphasis was to examine the behavior of the primary properties, such as velocity, density, pressure, and temperature, due to changes on the gap $L / H$ ratio. The analysis showed that for the conditions investigated, the gap flow behavior in the transition flow regime differed from that found in the continuum flow regime. It was found only one vortex for the $L / H$ ratio of $1,1 / 2,1 / 3$ and $1 / 4$. Conversely, in the continuum flow regime, the number of vortices inside the gap is approximately given by the amount $H / L$.

In continuation of the surface discontinuity study, Leite and Santos $[16,17]$ have investigated forward- and backward-facing steps situated in a rarefied hypersonic flow also by employing the DSMC method. The studies were motivated by the interest in examining the impact of the frontal- and back-face heights on the flowfield structure and on the aerodynamic surface properties in the transition flow regime, i.e., between the continuum flow and the free collision flow regime. Computational results for the forward-facing step showed that the step height changes contributed to significant modifications in the flowfield structure ahead the step. The analysis showed a dramatically increase in the primary flow properties at the vicinity of the step concave corner. Aerodynamic surface properties were directly influenced by the recirculation region ahead the step. Furthermore, high pressure and heat loads were observed at the vicinity of the step frontal face as a consequence of the recirculation region ahead the step.

Given the number of studies on cavities, gaps and steps, the study being reported herein is directed to expand the previous studies on surface discontinuities [15-17] by focusing on the more limited subject of surface discontinuity defined by the combination of a gap and a step. It is important to recall that this type of discontinuity, a gap/step configuration, may occur, for instance, in the windward surface of the Space Shuttle Orbiter. The windward surface is composed by a large number of thermal protection tiles. However, a misalignment between individual tiles may occur. Therefore, the misaligned tiles may constitute in a potential source in a heat flux to and pressure rise on the surface or even though in a premature transition from laminar to turbulent flow. In this scenario, the primary goal of this investigation is to provide a comprehensive description of the flowfield structure with special attention to the behavior 
of the primary properties, such as velocity, density, pressure, and temperature fields. The focus of the present work is on the low-density region in the upper atmosphere, where numerical gaskinetic procedures are available to simulate hypersonic flows. Therefore, the DSMC method will be employed to calculate the hypersonic two-dimensional flow on the gap/step configuration.

\section{COMPUTATIONAL TOOL}

The DSMC method is a powerful numerical technique pioneered by Bird [18]. The DSMC method is based on the physical concepts and physical assumptions that form the basis for the derivation of the Boltzmann equation. As a result, the DSMC method is subjected to the same restrictions as the Boltzmann equation. In this fashion, the DSMC method is subjected to the restriction to the dilute gases and to the assumption of molecular chaos.

Nowadays, the DSMC method has been recognized as an extremely powerful technique capable of predicting an almost unlimited variety of rarefied flowfields in the regimes where neither the Navier-Stokes nor the free molecular approaches are appropriate. The method has been thoroughly tested in high Knudsen number flows over the past 50 years and found to be in excellent agreement with experimental data [19-21]. Comparisons with experiments have given credibility and have been vital in gaining widespread acceptance of the method.

The DSMC technique reproduces the physics of the Boltzmann equation by following the motions and collisions of a large number of modeling molecules. Each modeling molecule possesses a position, velocity, and internal energy. The state of the molecules is stored and modified with time as the molecules move, collide, and undergo boundary interactions in simulated physical space. The assumption of a dilute gas, which means that the mean molecular diameter is much less than the mean molecular spacing of molecules in the gas, allows for the molecular motion to be decoupled from the molecular collisions [18]. Particle motions are modeled deterministically, while the collisions are treated statistically. Since it is impossible to simulate the real number of molecules in the computational domain, a small number of representative molecules is used, where each representative molecule corresponds to a large number of real molecules.

The study at hand employs a DSMC code developed in the Brazilian Institute for Space Research (INPE) based on the traditional DSMC code [18]. In this code, intermolecular collisions are treated by using the variable hard sphere (VHS) molecular model [22], and the no time counter (NTC) collision sampling technique [23]. The VHS model employs the simple hard sphere angular scattering law so that all directions are equally possible for postcollision velocity in the center-of-mass frame of reference. Nevertheless, the collision cross section depends on the relative speed of colliding molecules. Simulations are performed by using a nonreacting gas model, consisting of $76.3 \%$ of $\mathrm{N}_{2}$ and $23.7 \%$ of $\mathrm{O}_{2}$, 
while considering energy exchange between translational, rotational, and vibrational modes. Energy partitioning is accounted for using the Borgnakke-Larsen statistical model [24] with rotational and vibrational collision numbers for internal relaxation, obtained in a collision energy-based procedure as suggested by Boyd [25] for rotation and by Bird [26] for vibration. For a given collision, the probabilities are designated by the inverse of the relaxation numbers, which correspond to the number of collisions necessary, on average, for a molecule to relax.

\section{GEOMETRY DEFINITION}

In the present calculations, the discontinuities on the surface of a reentry vehicle are modeled by a flat plate with a gap of length $L$ and depth $H$ and a forwardfacing step with height $h$. The combination of a gap with a forward-facing step is defined herein as a gap/step configuration. Figure $1 a$ displays a schematic view of the model employed. The flat plate was selected by considering that the gap depth $H$ or the step height $h$ is much smaller than the nose radius $R$ of a reentry vehicle, i.e., $H / R \ll 1$ or $h / R \ll 1$. Therefore, the hypersonic flow over the flat plate with a gap/step may be representative of the hypersonic flow over a gap/step configuration located on the surface of a reentry vehicle.

According to Fig. $1 a, \mathrm{M}_{\infty}$ represents the free-stream Mach number, $H$ is the gap depth, $h$ is the step height, $L_{u}$ is the length of the gap/step upstream surface, $L$ is the length of the gap, and $L_{d}$ is the length of the gap/step downstream surface. It was assumed a gap length $L$ of $3 \mathrm{~mm}$ and a gap depth $H$ of $12 \mathrm{~mm}$, which correspond to a length-to-depth ratio, $L / H$, of $1 / 4$. In addition, the step height $h$ investigated was defined as being 3,6 , and $9 \mathrm{~mm}$, which correspond to the dimensionless height $h^{*}\left(\equiv h / \lambda_{\infty}\right)$ of $3.23,6.46$, and 9.69 , respectively, where

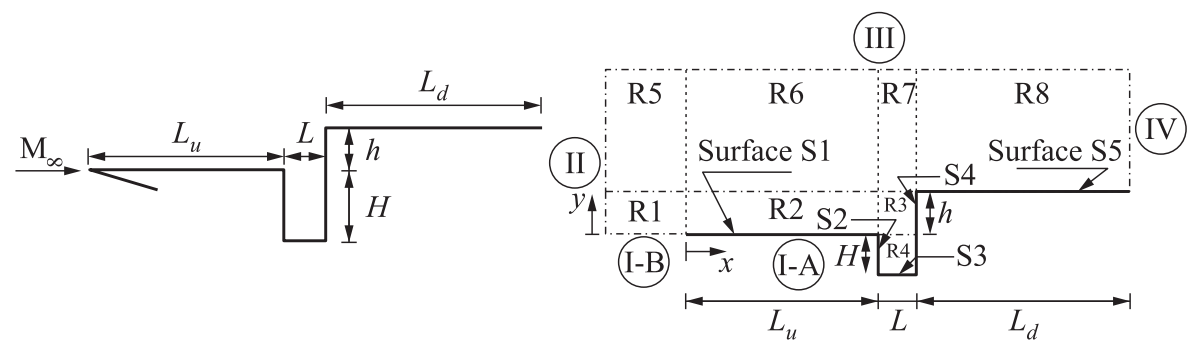

(a)

(b)

Figure 1 Illustrations of the gap/step configuration $(a)$ and the computational domain (b) 
$\lambda_{\infty}$ is the free-stream mean free path. Furthermore, it was defined $L_{u} / \lambda_{\infty}$ of 50 and $L_{d} / \lambda_{\infty}$ of 50 . It was considered that the flat plate is infinitely long but only the total length $L_{u}+L+L_{d}$ is investigated.

\section{FREE-STREAM AND FLOW CONDITIONS}

Free-stream flow conditions employed in the present calculations are those given by Leite and Santos [16] in the previous work and summarized in Table 1. These free-stream conditions represent those experienced by a reentry vehicle at an altitude of $70 \mathrm{~km}$. Based on Table $1, T_{\infty}, p_{\infty}, \rho_{\infty}, \mu_{\infty}, n_{\infty}, \lambda_{\infty}$, and $U_{\infty}$ stand, respectively, for temperature, pressure, density, viscosity, number density, mean free path, and velocity. In addition, the gas properties [18] are shown in Table 2. According to Table 2, $\chi, m, d$, and $\omega$ account, respectively, for mole fraction, molecular mass, molecular diameter, and viscosity index.

The free-stream velocity $U_{\infty}$, assumed to be constant at $7546.5 \mathrm{~m} / \mathrm{s}$, corresponds to a free-stream Mach number $\mathrm{M}_{\infty}$ of 25 . The wall temperature $T_{w}$ is assumed constant at $880 \mathrm{~K}$. This temperature is chosen to be representative of the surface temperature near the stagnation point of a reentry capsule, and it is assumed to be uniform on the gap/step surface. It is important to mention that this surface temperature is low compared to the free-stream stagnation temperature. This assumption seems reasonable since practical surface material will probably be destroyed if surface temperature is allowed to approach the stagnation temperature.

By assuming the step frontal-face height $h$ as the characteristic length, the Knudsen number $\mathrm{Kn}_{h}$ corresponds to 0.3095 , 0.1548, and 0.1032 for step heights $h^{*}$ of $3.23,6.46$, and 9.69 , respectively. Finally, the Reynolds number $\mathrm{Re}_{h}$, also based on the frontal-face height $h$ and on the conditions in the undisturbed stream, is around 136, 272, and 409 for the same step heights $h^{*}$ $(3.23,6.46$, and 9.69 , respectively).

Table 1 Free-stream flow conditions

\begin{tabular}{ccccccc}
\hline$T_{\infty}, \mathrm{K}$ & $p_{\infty}, \mathrm{N} / \mathrm{m}^{2}$ & $\rho_{\infty}, \mathrm{kg} / \mathrm{m}^{3}$ & $\mu_{\infty}, \mathrm{N} \cdot \mathrm{s} / \mathrm{m}^{2}$ & $n_{\infty}, \mathrm{m}^{-3}$ & $\lambda_{\infty}, \mathrm{m}$ & $U_{\infty}, \mathrm{m} / \mathrm{s}$ \\
\hline 219.69 & 5.582 & $8.753 \cdot 10^{-5}$ & $1.455 \cdot 10^{-5}$ & $1.8192 \cdot 10^{21}$ & $9.285 \cdot 10^{-4}$ & 7546.5 \\
\hline
\end{tabular}

Table 2 Gas properties

\begin{tabular}{ccccc}
\hline Gas & $\chi$ & $m, \mathrm{~kg}$ & $d, \mathrm{~m}$ & $\omega$ \\
\hline $\mathrm{O}_{2}$ & 0.237 & $5.312 \cdot 10^{-26}$ & $4.07 \cdot 10^{-10}$ & 0.77 \\
$\mathrm{~N}_{2}$ & 0.763 & $4.650 \cdot 10^{-26}$ & $4.17 \cdot 10^{-10}$ & 0.74 \\
\hline
\end{tabular}




\section{COMPUTATIONAL FLOW DOMAIN AND GRID}

In order to implement the particle-particle collisions, the flowfield around the gap/step configuration is divided into an arbitrary number of regions, which are subdivided into computational cells. The cells are further subdivided into subcells, two subcells/cell in each coordinate direction. The cell provides a reference for the sampling of the macroscopic gas properties, such as density, velocity, pressure, temperature, etc., while the subcell is used in order to select collision partners for the establishment of the collision rate. Therefore, the physical space network is used to facilitate the choice of molecules for collisions and for the sampling of the macroscopic flow properties.

The computational domain used for the simulation is made large enough so that disturbances from the gap/step configuration do not reach the upstream and side boundaries, where free-stream conditions are specified. A schematic view of the computational domain is demonstrated in Fig. 1b. According to this figure, side I-A is defined by the gap/step surface. Diffuse reflection with complete thermal accommodation was defined as the condition applied to this side. In a diffuse reflection, molecules are reflected equally in all directions, and the final velocity of the molecules is randomly assigned according to a half-range Maxwellian distribution determined by the wall temperature. In addition, the internal energies, rotation and vibration, of the molecules are also sampled from the appropriate equilibrium distribution by using the wall temperature. Side I-B represents a plane of symmetry, where all flow gradients normal to the plane are zero. At the molecular level, this plane is equivalent to a specular reflecting boundary. For a specular reflection, the only change to the properties of the molecules is the velocity component normal to the surface that is simply reversed in sign. Sides II and III are the free-stream sides through which simulated molecules enter and exit. Side II is positioned at $5 \lambda_{\infty}$ upstream the flat-plate leading edge and side III is defined at $30 \lambda_{\infty}, 34 \lambda_{\infty}$, and $42 \lambda_{\infty}$ above surface S5 (see Fig. $1 b$ ) for frontal-face height $h^{*}$ of $3.23,6.46$, and 9.69 , respectively. Finally, the flow at the downstream outflow boundary, side IV, is predominantly supersonic and vacuum condition is specified [18]. Basically, at this boundary, simulated molecules can only exit. Nevertheless, it is important to mention that, close to the wall, molecules may not be moving at supersonic speed. As a result, in this subsonic region close to the wall, there is an interaction between the flow and the downstream boundary. However, the extent of the upstream effect of this boundary condition can be determined by changing the length of surface S5. For the conditions investigated in the present calculations, the upstream disturbance is approximately of $6 \lambda_{\infty}[16]$.

The DSMC results depend on the cell size chosen, on the time step as well as on the number of particles per computational cell. The linear dimensions of the cells should be small in comparison with the length scale of the macroscopic flow gradients normal to the streamwise directions, which means that the cell 


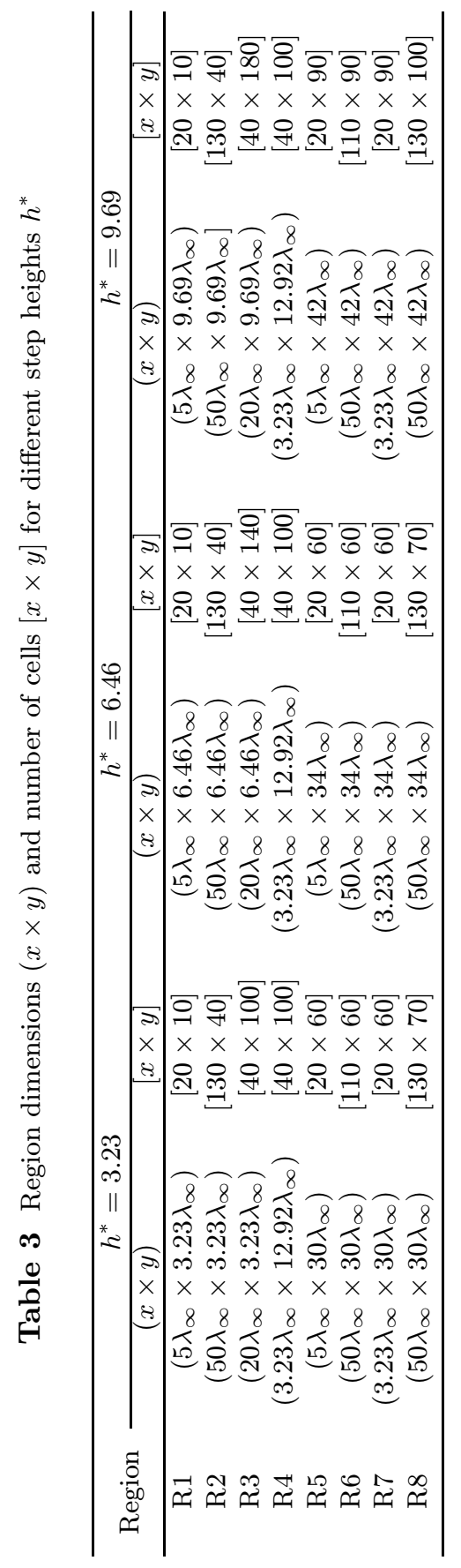


dimensions should be of the order or smaller than the local mean free path $[27,28]$. The time step should be chosen to be sufficiently small in comparison with the local mean collision time $[29,30]$. A very small time step results in an inefficient advancement of the solution and accumulation of statistics. Most particles will take many time steps to cross a given cell. As a result, the collision phase of each time step will involve the same group of particles as in the previous time step since almost no particles leave or enter the cell. Moreover, a large time step allows the molecules to move too far without the opportunity to participate in a collision. This again causes a smearing of the properties of the flow, resulting in nonphysical results. Therefore, the time step must be chosen such that a typical particle moves about one fourth of the cell dimension at each time step. Finally, the number of simulated particles has to be large enough to make statistical correlations between particles insignificant. It is advisable that each cell be populated with a minimum number of molecules, typically around $20[31,32]$.

A grid independence study was made with three different structured meshes - coarse, standard, and fine - in each coordinate direction. The effect of altering the cell size in the $x$ - and $y$-directions was investigated for a coarse and fine grids with, respectively, $50 \%$ less and $100 \%$ more cells with respect to the standard grid. Table 3 summarizes the main characteristics for the standard grid related to eight regions (R1 to R8 in Fig. $1 b$ ) for the frontal-face height $h^{*}$ of $3.23,6.46$, and 9.69 . So, for $h^{*}$ of $3.23,6.46$, and 9.69 , the total number of cells correspond, respectively, to 31500,33100 , and 43100 cells.

The effect (not shown) of changing the cell size in both $x$ - and $y$-directions on the heat transfer, pressure, and skin friction coefficients was rather insensitive to the range of cell spacing considered, indicating that the standard grids, with a total of 31500,33100 , and 43100 cells are essentially grid-independent.

\section{COMPUTATIONAL RESULTS AND DISCUSSION}

This section focuses on the effects that take place in the flowfield structure due to variations on the step frontal-face height of a gap/step configuration. Therefore, the purpose of this section is to discuss and to compare the differences in the flowfield properties due to variations on the step frontal-face height. Flowfield properties of particular interest in this work are velocity, density, pressure, and kinetic temperatures.

Before proceeding with the analysis of the flowfield properties, it proves instructive to present first the flow topology inside a gap (without a step) and around a forward-facing step (without a gap), as investigated previously by Paolicchi and Santos [15] and Leite and Santos [16], respectively. In doing so, streamline traces inside a gap with a $L / H$ of $1 / 4$ and around a forward-facing step with $h^{*}$ of 3.23 and 9.69 are depicted in Fig. 2. The flow topology for the $h^{*}=6.46$ case is intermediate to the other two cases, and it will not be shown. 


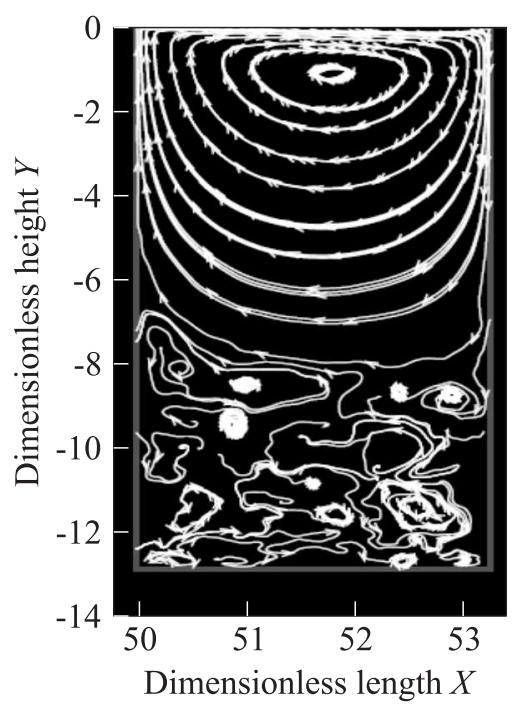

(a)

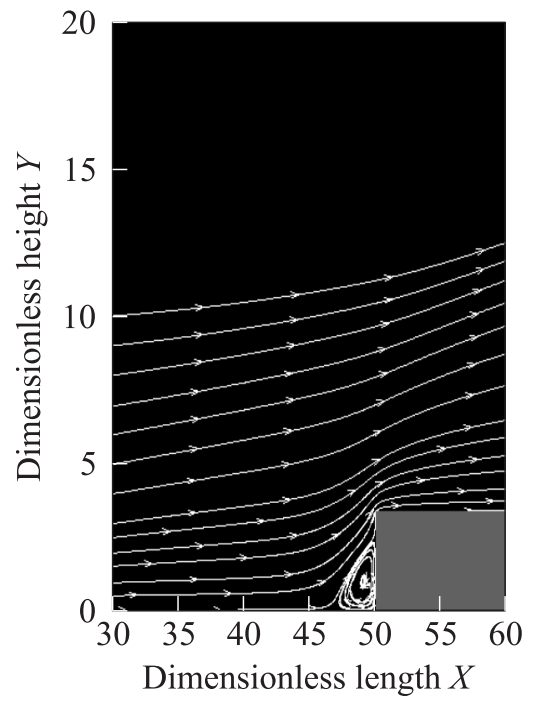

(b)

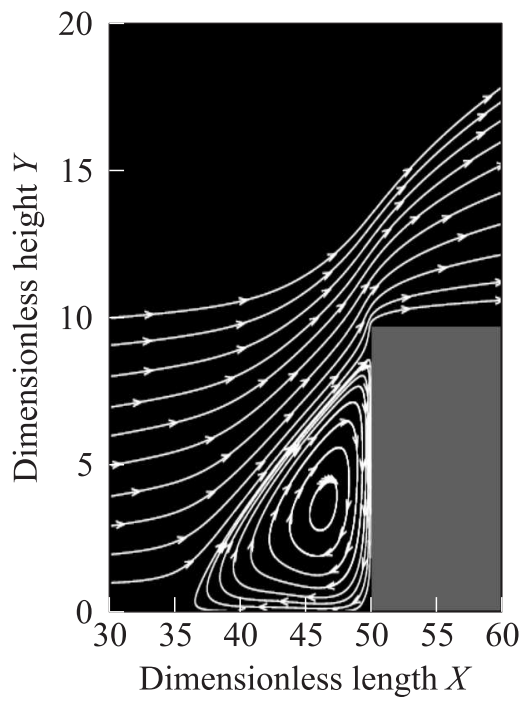

(c)

Figure 2 Distribution of streamline traces inside the gap with $L / H$ of $1 / 4(a)$ and around the step with frontal-face thickness $h^{*}$ of $3.23(b)$ and $9.69(c)$ 
In this set of plots, $X$ represents the distance $x$ from the sharp leading edge normalized by $\lambda_{\infty}$, and $Y$ the distance $y$ above the surface also normalized by $\lambda_{\infty}$. For convenience and later reference, the gap case corresponds to the geometry illustrated in Fig. $1 b$ with $h=0$ and the forward-facing step corresponds to $L=0$.

Looking first at Fig. $2 a$, it is clearly seen that flow within the gap, defined by $L / H$ ratio of $1 / 4$, is characterized by a primary vortex system. In addition, the recirculation region do not fill the entire gap, and the external stream does not reattach at the bottom surface of the gap. Furthermore, the flow exhibits an irregular or chaotic structural motion in the second half part of the gap, more precisely, for $Y<-7$. In this context, it should be mentioned that the gap flow topology observed here in a rarefied environment differs from that usually observed in the continuum flow regime. In the continuum flow regime, the gap flow topology is defined by the development of a column of counterrotating vortices within the gap caused by the main stream flow, where the number of vortices is approximately given by $H / L$. In addition, alternating hot spots are developed in the gap when the vortices directionally align and impinge on the gap sidewall [33].

Turning next to Figs. $2 b$ and $2 c$, it is clearly noticed that a recirculation region appears at the vicinity of the frontal face of the steps. It may be inferred by visual inspection that the recirculation region increases with increasing the step height $h^{*}$ or by increasing the Reynolds number $\mathrm{Re}_{h}$ not only along the upstream surface but also along the frontal-face surface. This behavior differs from that one observed in a continuum flow regime. Based on an experimental investigation, Camussi et al. [34] pointed out that the separation region occurs independently of the Reynolds number $\mathrm{Re}_{h}$. They found that the separation region is of the order of $h$ along upstream surface and approximately a half $h$ size along the step-frontal-face surface.

\subsection{Velocity Field}

The distribution of tangential velocity $u$ for three sections along the gap/step upstream surface, surface S1, is illustrated in Fig. 3 as a function of the step frontal-face height $h^{*}$. In this set of plots, velocity ratio is the tangential velocity $u$ normalized by the free-stream velocity $U_{\infty}, X$ represents the distance $x$ from the sharp leading edge normalized by $\lambda_{\infty}$, and $Y$ the distance $y$ above surface S1 also normalized by $\lambda_{\infty}$. In addition, filled and empty symbols correspond to step height $h^{*}$ of 3.23 and 9.69 , respectively. As a basis of comparison, the tangential velocity profiles for the flat-plate case, for the gap and for the forward-facing step investigated separately in previous studies $[15,16]$ are also illustrated in the same set of plots. It is important to mention that tangential velocity profiles for the $h^{*}=6.46$ case are intermediate to the other two cases; therefore, they will not be shown. 


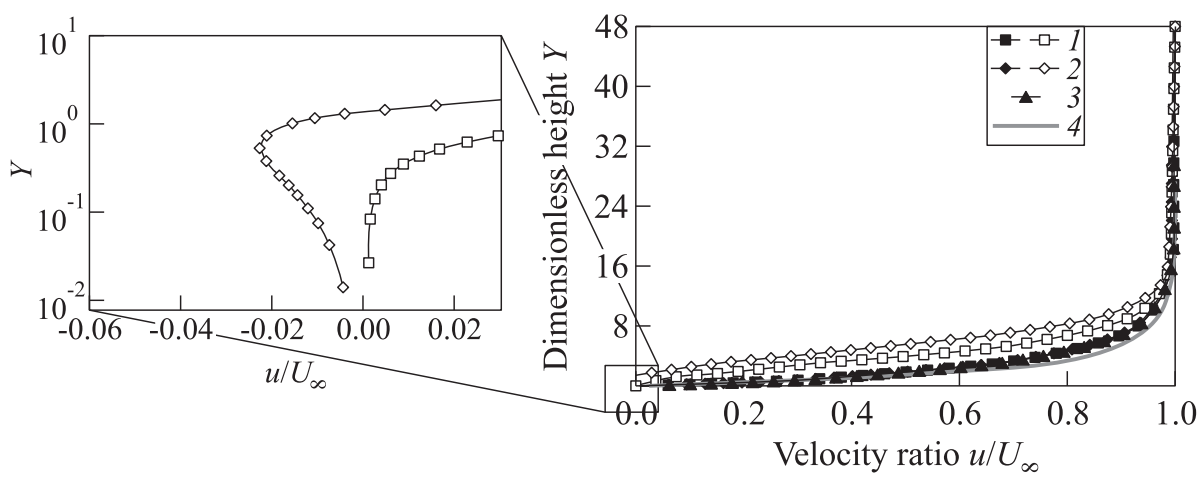

(a)

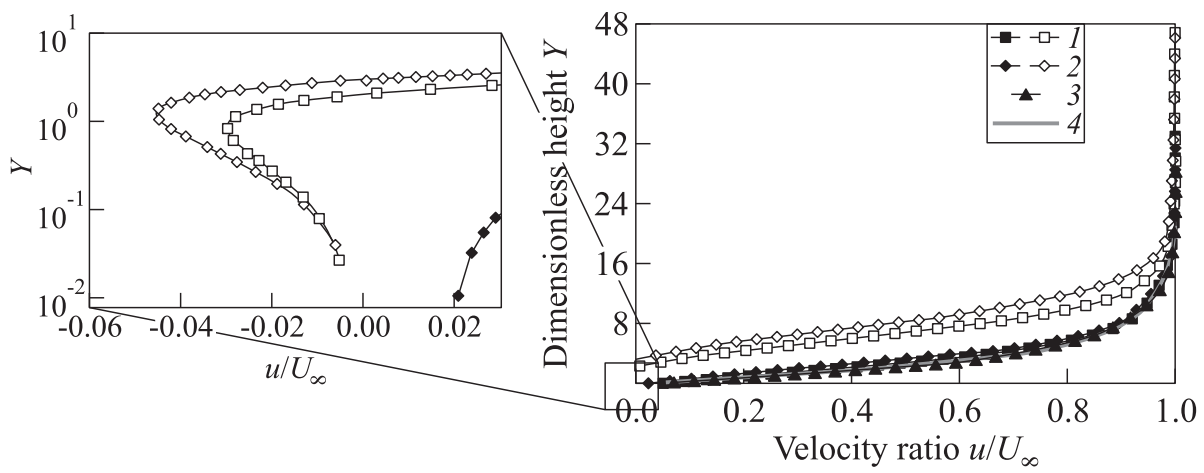

(b)

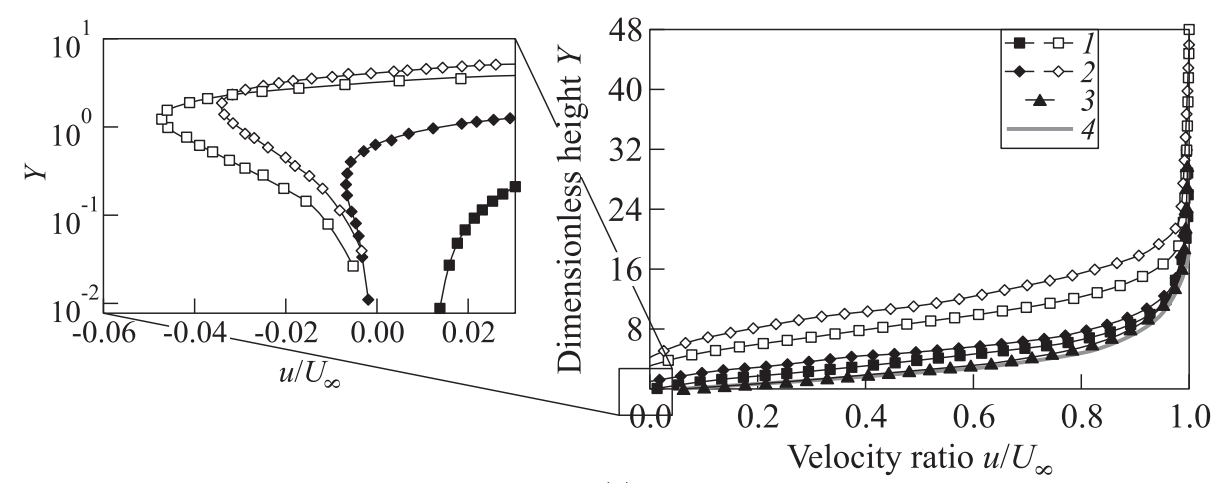

(c)

Figure 3 Distribution of tangential velocity $\left(u / U_{\infty}\right)$ for three sections on surface S1 of the gap/step configurations ( 1 - gap/step; 2 - forward-facing step; and 3 - gap) defined by section $X$ of $39(a), 44(b)$, and $48(c)$. Filled symbols refer to $h^{*}=3.23$; empty symbols refer to $h^{*}=9.69 ; 4-$ flat-plate case 
Important features can be observed in the profiles of tangential velocity shown in this set of plots. For section $X=39$, the velocity profiles for the gap, step, and gap/step configuration, defined by $h^{*}=3.23$, are basically identical, by visual inspection, to those for the flat-plate case. It means that no effect of the presence of the step or the gap is observed up to this section. Conversely, for the $h^{*}$ $=9.69$ case, the presence of the gap/step configuration is felt far upstream along surface S1. Moreover, for sections $X \geq 44$, the upstream disturbance caused by the forward-facing steps or by the gap/step configurations is observed on the velocity profiles for the frontal-face height $h^{*}$ investigated. In addition, it is observed that the velocity profile is affected more upstream with increasing the frontal-face height $h^{*}$. This behavior results from the diffusion of molecules that are reflected from the frontal face of the step. Also, for sections $X$ $\geq 44$, the velocity profiles related to the forward-facing steps and some gap/step configurations indicate negative velocities close to surface S1, characterizing a recirculation region at the vicinity of the frontal face. For section $X=39$, negative velocity occurs only for the $h^{*}=9.69$ case. Moreover, for section $X=48$, negative velocity occurs for the three frontal-face heights investigated for the forward-facing step, while for the gap/step configuration, negative velocity is not observed for the $h^{*}=3.23$ case, as displayed by the magnified view shown in Fig. $3 c$. It is perhaps worth noting that in previous investigations $[15,16]$, steps and the gaps were placed at section $X=50$.

Still referring to Fig. 3, it is firmly established that the tangential velocity profiles for the gap are identical to those for the flat-plate case, indicating that the presence of the gap (without a step) does not influence the flowfield upstream the gap. In contrast, the presence of a step affected the flowfield far upstream, as was expected. Moreover, the effect of the gap/step combination was less intense than that observed for the step alone. In an effort to emphasize the points of interest related to the velocity field, Fig. 4 displays Mach number contour maps along with streamline traces inside the gap/step configuration for $h^{*}$ of 3.23 , 6.46, and 9.69. It may be recognized from this set of plots that, in general, the gap/step configuration presents a recirculation region inside the gap and another one outside the gap, i. e., ahead of the step frontal face. It is seen that, for the recirculation region ahead of the step, the flow goes in the counterclockwise direction. Conversely, inside the gap, the flow goes in the clockwise direction. This behavior is in agreement with that illustrated in Fig. 2, i.e., when the flowfield structure for gap and step is investigated separately. Of particular interest is the recirculation region for the $h^{*}=3.23$ case, displayed in Fig. $4 a$. It seems that the primary vortex, initially inside the gap, moves outside the gap with increasing the step frontal face. Afterwards, another vortex is formed inside the gap, as demonstrated in Figs. $4 b$ and $4 c$.

As indeed is clear from Fig. 4, after the flow separation on the lower surface, the flow reattaches on the frontal face at the vicinity of the step shoulder. Table 4 tabulates the separation point $x_{s}$ and the reattachment point $y_{r}$ as a function 


\section{Mach number}

$\begin{array}{llllllllllllllllllll}1 & 2 & 4 & 5 & 6 & 7 & 9 & 10 & 11 & 12 & 14 & 15 & 16 & 17 & 19 & 20 & 21 & 22 & 24 & 25\end{array}$

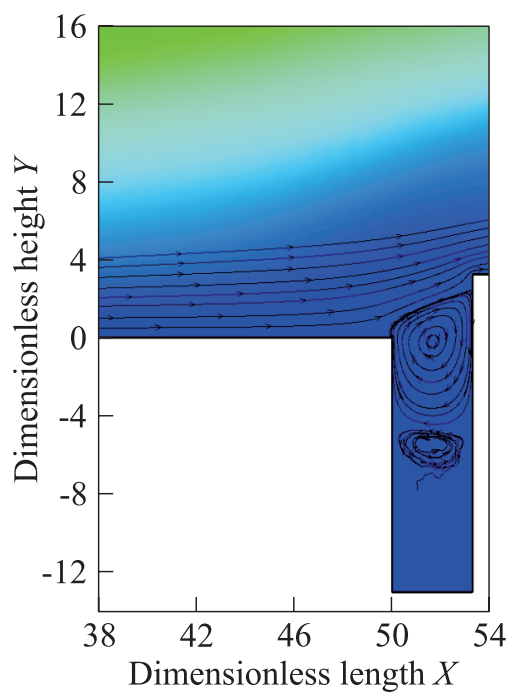

(a)

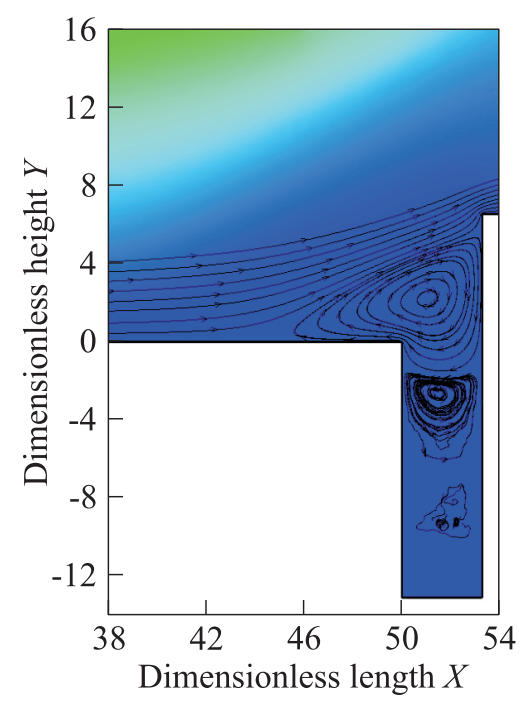

(b)

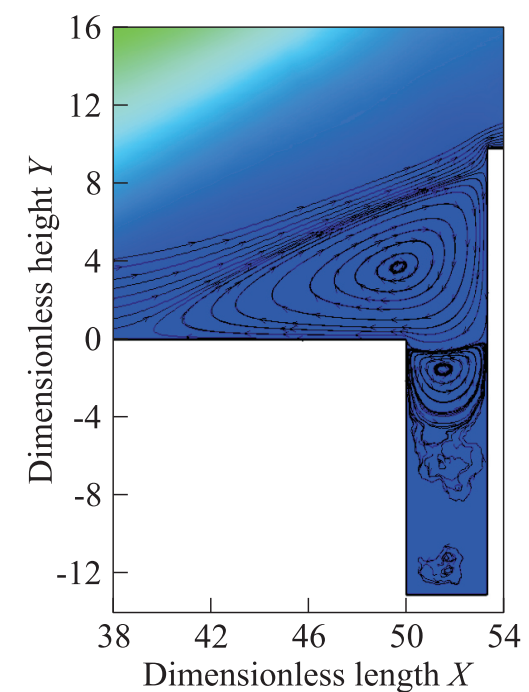

(c)

Figure 4 Mach number contour maps along with streamline traces at the vicinity of the gap/step configuration for step height $h^{*}$ of $3.23(a), 6.46(b)$, and $9.69(c)$; $L / H=1 / 4$ 
Table 4 Separation and reattachment points for the gap/step configuration as a function of the dimensionless frontal-face height $h^{*}$

\begin{tabular}{cccccc}
\hline$h^{*}$ & $X_{s}$ & $X_{s h}$ & $X_{s h}^{\prime}$ & $Y_{r}$ & $Y_{r h}$ \\
\hline 3.23 & $50.00[48.69]$ & $15.48[15.07]$ & $-1.00[-0.41]$ & $2.73[2.72]$ & $0.84[0.84]$ \\
6.46 & $46.38[42.99]$ & $7.18[6.65]$ & $-1.06[-1.09]$ & $5.60[5.61]$ & $0.87[0.87]$ \\
9.69 & $40.62[37.42]$ & $4.19[3.86]$ & $-1.30[-1.30]$ & $8.68[8.66]$ & $0.90[0.89]$ \\
\hline
\end{tabular}

of the dimensionless frontal-face height $h^{*}$ for the gap/step configuration. In this table, $X_{s}$ and $X_{s h}$ represent, respectively, the separation point $x_{s}$ normalized by the free-stream mean free path $\lambda_{\infty}$ and by the step height $h$. Also, $X_{s h}^{\prime}$ correspond to $\left(x_{s}-L_{u}-L\right)$ normalized by the step height $h$. Similarly, $Y_{r}$ and $Y_{r h}$ stand for the point $y_{r}$ normalized by $\lambda_{\infty}$ and $h$, respectively. For comparison purpose, the results for the forward-facing step cases [16] are shown inside brackets. According to this table, it is noticed that, in general, the separation points for the gap/step configuration occurred further downstream along surface S1 (approximately, a gap length $L$ ) as compared to those for the forwardfacing step cases. Nevertheless, for the conditions investigated, the recirculation region for the gap/step configuration basically presents the same size of that for the forward-facing step. In addition, similar to the forward-facing step, the separation and reattachment points for the gap/step configuration change with increasing the step frontal-face height $h^{*}$.

Still referring to Table 4, a feature of particular interest is related to the gap/step configuration defined by $h^{*}=3.23$ case. For this particular case, the separation occurred at the convex corner, at section $X=50$, i. e., at the surface$\mathrm{S} 1 /$ surface-S2 junction. It is noteworthy that, according to Fig. 3, there were not observed negative velocities along surface S1 for the $h^{*}=3.23$ case, an indication that the flow did not change its direction close to surface S1.

It is important to recall that the separation point $x_{s}$ on surface S1 and the reattachment point $y_{r}$ on the frontal face were obtained on the basis of zero skin friction coefficient, $C_{f}=0$ (or wall shear stress $\tau_{w}=0$ ). The reason is that the skin friction coefficient along a surface usually changes from positive to negative value at separation and vice versa at reattachment, as pointed out by Kim and Setoguchi [35] and Deepak et al. [36]. Therefore, it is a good indication of the position of separation at surface S1 and reattachment at the frontal face.

\subsection{Density Field}

The distribution of density $\rho$ for three sections along surface S1 of the gap/step configuration is displayed in Fig. 5 (left column), parameterized by the dimen- 

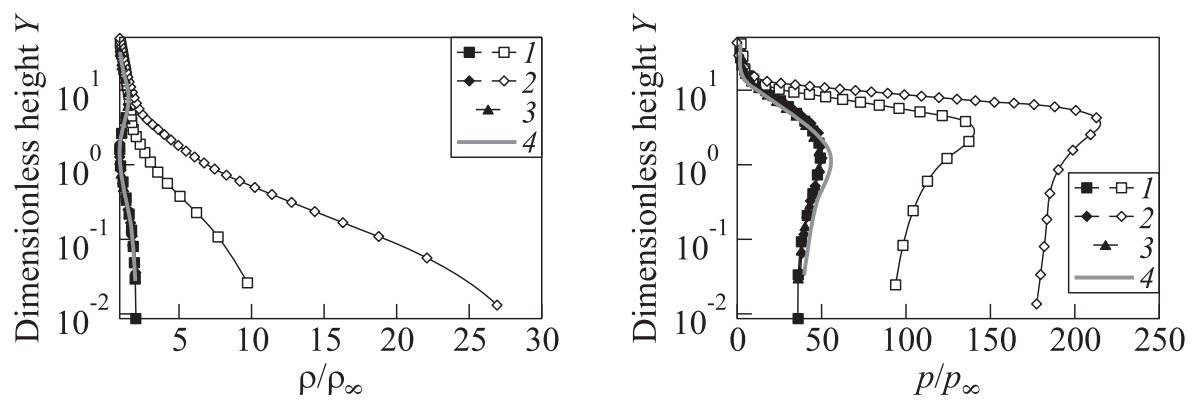

(a)
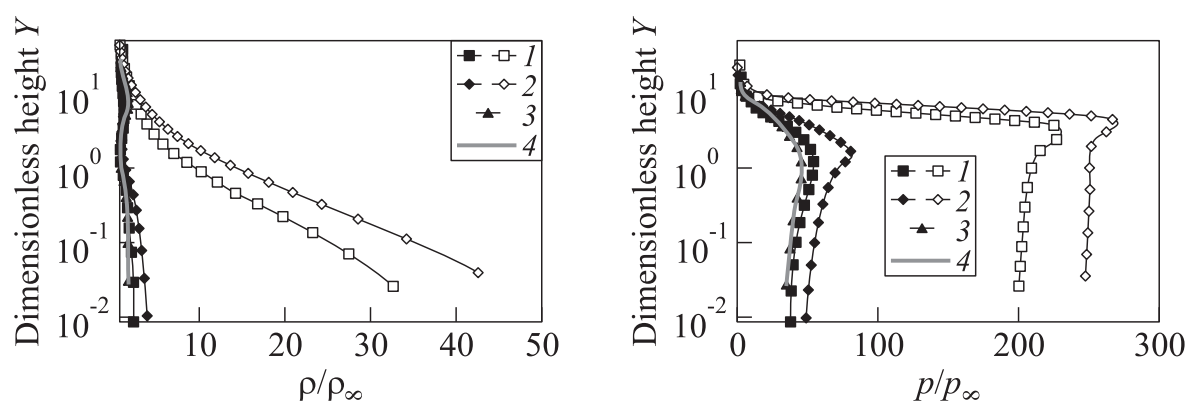

(b)
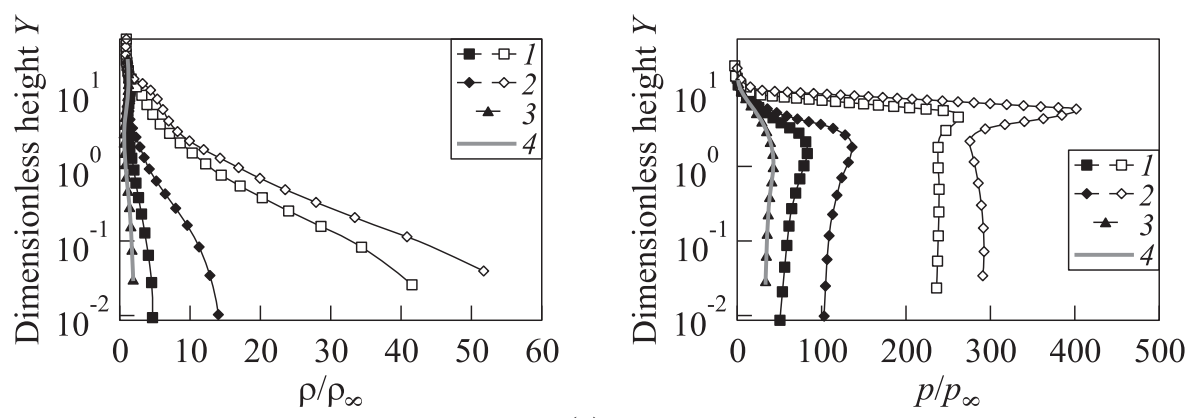

(c)

Figure 5 Density ratio $\rho / \rho_{\infty}$ (left column) and pressure ratio $p / p_{\infty}$ (right column) profiles for three sections on surface S1 of the gap/step configurations (1 - gap/step, 2 - forward-facing step; and 3-gap) defined by section $X$ of $39(a), 44(b)$, and $48(c)$. Filled symbols refer to $h^{*}=3.23$; empty symbols refer to $h^{*}=9.69 ; 4$ - flat-plate case 
sionless step height $h^{*}$. Similar to tangential velocity profiles, density profiles are presented for three sections defined by $X=39,44$, and 48 . In this group of plots, density ratio refers to density $\rho$ normalized by the free-stream density $\rho_{\infty}$. Again, $X$ represents the distance $x$ normalized by the free-stream mean free path $\lambda_{\infty}$ and $Y$ the distance $y$ above surface S1 also normalized by $\lambda_{\infty}$. For comparison purpose, density profiles for the flat-plate case, for the gap, and for the forward-facing step are also presented in the same group of plots. Due to the large range of variation for the ratio $\rho / \rho_{\infty}$ along surface $\mathrm{S} 1$, the scale in the $x$-direction may differ from one plot to another.

According to Fig. 5 (left column), it is observed that the upstream disturbance imposed by the step and by the gap/step configuration, for the $h^{*}=9.69$ case, influences the density profile at section $X=39$ (see Fig. $5 a$, left column). In contrast, there is no indication that density profile is affected by the presence of the step or by the gap/step configuration for the $h^{*}=3.23$ case. As expected, by increasing the frontal face $h$, the disturbance caused by the step influences more upstream in the flow. It should be mentioned in this context that for $X<28$, the density profiles (not shown) for the step cases are identical to those for the flat-plate case.

Another flow peculiarity is observed in Fig. 5 (left column). It is noted that density dramatically increases as the flow approaches the step frontal face, i. e., the density $\rho$ increased at least by one order of magnitude when compared to the free-stream density $\rho_{\infty}$, as illustrated in the profiles for section $X=48$ (see Fig. $5 c$ ). For comparison purpose, the density ratio $\rho / \rho_{\infty}$ for step cases is around $32.9,56.2$, and 72.3 for $h^{*}$ of $3.23,6.46$, and 9.69 , respectively, at the step base [37]. On the other hand, the density ratio for the gap/step configuration at the vicinity of the frontal face is smaller than that for the forward-facing step (without gap). The reason for that is because at the vicinity of the frontal face, the flow enters into the gap. As a result, for the gap/step configuration, the density inside the gap increases dramatically as compared to the density observed in the gap (without a step). As a base of comparison, for the gap with an $L / H$ of $1 / 4$, as that illustrated in Fig. $2 a$, the density inside the gap is around three times the free-stream density, for the same flow conditions [38].

Finally, in attempting to bring out the essential features of the density behavior inside the gap/step configuration, Fig. 6 (left column ) depicts the contours maps for the density ratio distribution in the vicinity of the gap/step configuration as a function of the frontal-face height $h^{*}$. In this group of contour maps, again dimensionless height $Y$ stands for the height $y$ normalized by the freestream mean free path $\lambda_{\infty}$ and the dimensionless length $X$ refers to the length $x$ also normalized by $\lambda_{\infty}$. From this set of plots, it may be recognized that density ratio increases inside the gap by increasing the frontal-face height $h^{*}$ for the conditions investigated. This is in contrast to the gap (without step) investigated previously. Consequently, the step frontal face acts as a barrier in the sense that part of the flow enters into the gap. 

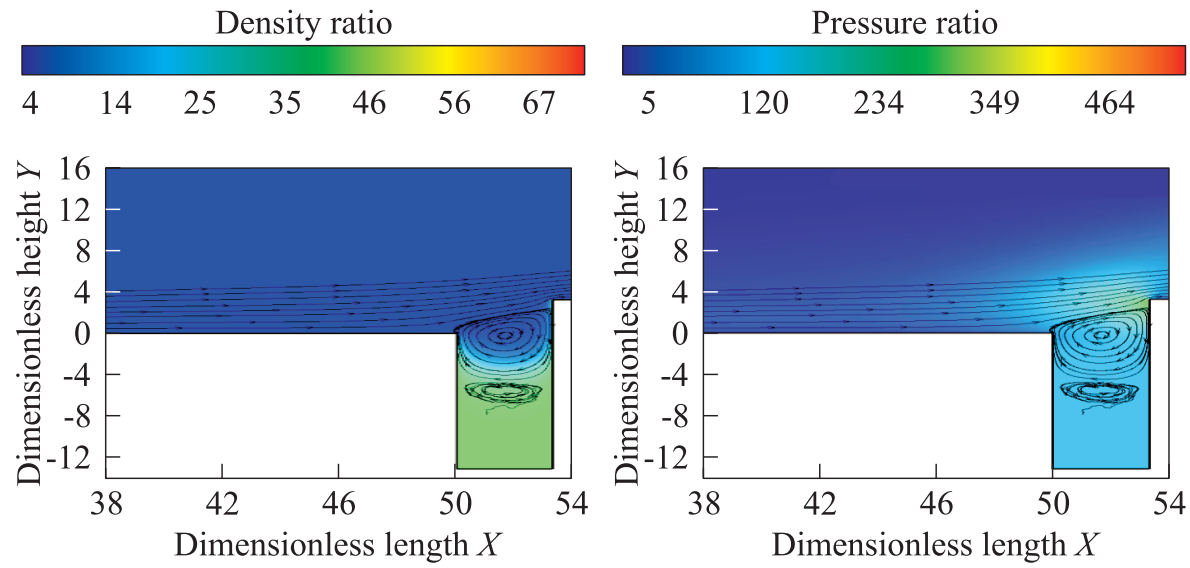

(a)
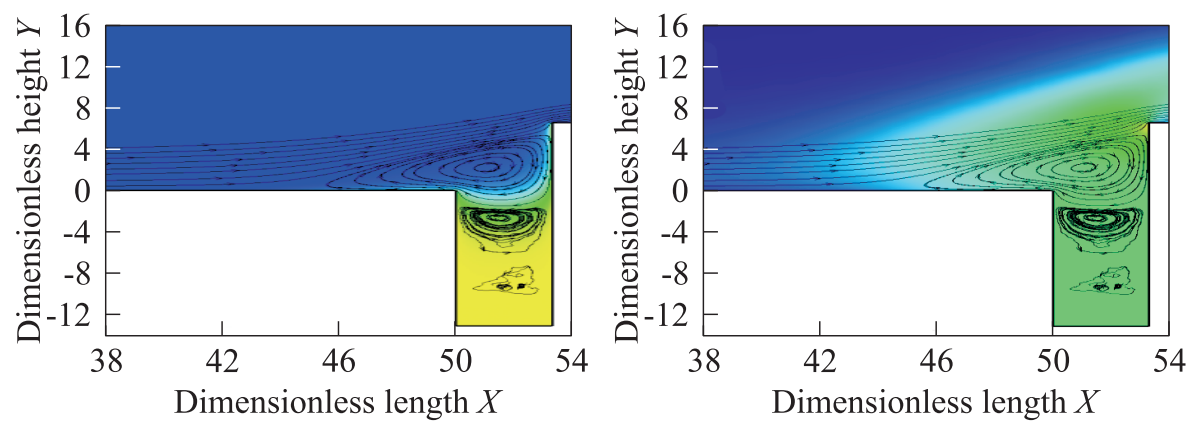

(b)
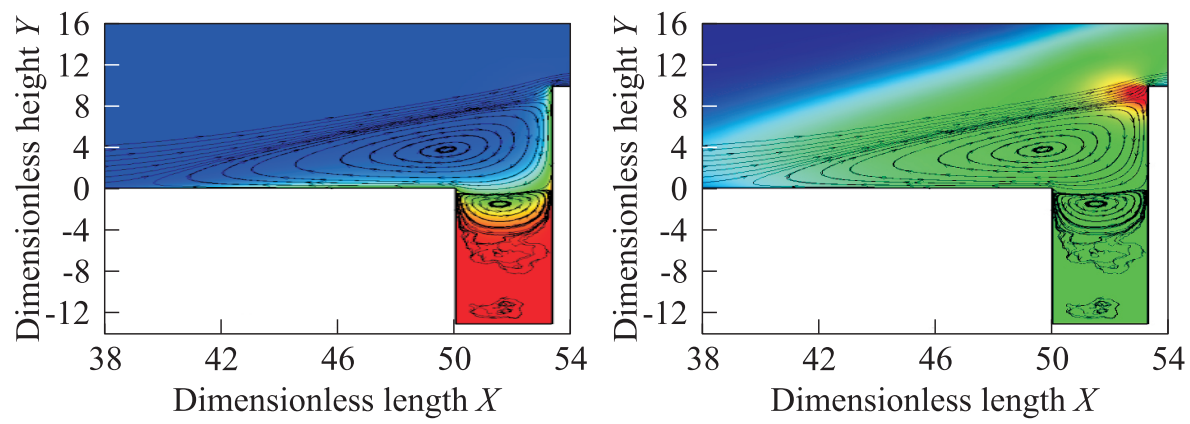

(c)

Figure 6 Density ratio $\rho / \rho_{\infty}$ (left column) and pressure ratio $p / p_{\infty}$ (right column) contour maps along with streamline traces at the vicinity of the gap/step configuration for step height $h^{*}$ of $3.23(a), 6.46(b)$, and $9.69(c) ; L / H=1 / 4$ 


\subsection{Pressure Field}

The distribution of pressure $p$ for three sections along surface S1 of the gap/step configuration is depicted in Fig. 5 (right column) as a function of the dimensionless step height $h^{*}$. In this set of diagrams, pressure ratio corresponds to pressure $p$ normalized by the free-stream pressure $p_{\infty} ; X$ and $Y$ are the dimensionless variables as defined previously in the density profiles. Again, as a matter of comparison, pressure profiles for the flat-plate, gap, and forward-facing step cases are also exhibited in these plots. It is important to emphasize that due to the large range of variation for the ratio $p / p_{\infty}$ along surface $\mathrm{S} 1$, the scale in the $x$-direction may differ from one plot to another.

Focusing on Fig. 5 (right column), it is noticed that pressure profiles follow a similar behavior as that presented by density profiles in the sense that pressure profiles for the gap, forward-facing step, and gap/step, particularly, for the $h^{*}=3.23$ case, are basically identical to that for the flat-plate case at section $X=39$ (see Fig. $5 a$, right column). This is an indication that pressure field has not been affected yet by the presence of the gap, step, or the gap/step configuration. However, by increasing the frontal face $h^{*}$, for instance, from 3.23 to 9.69 , the upstream disturbance increases not only for the forward-facing step but also for the gap/step configuration. As the flow approaches the frontal face, at section $X=44$ (see Fig. $5 b$, right column), the pressure field is already affected by the presence of the forward-facing step for the $h^{*}=3.23$ case. In contrast, no effect is observed yet in the pressure profile due to the presence of the gap/step configuration for the $h^{*}=3.23$ case. It may be inferred that the presence of the gap in the gap/step configuration attenuates the upstream disturbances. In the following, at section $X=48$ (see Fig. $5 c$, right column), pressure profiles for the step cases and for the gap/step cases differ considerably from those for the flat-plate case. It is also noticed that pressure ratio dramatically increases as the flow approaches the step frontal face. It is seen that pressure $p$ increased two orders of magnitude as compared to the free-stream pressure $p_{\infty}$. As an illustrative example, for the forward-facing step, at the step base, the pressure ratios $p / p_{\infty}$ are 142, 230, and 293 for heights $h^{*}$ of 3.23, 4.46, and 9.69, respectively. Consequently, particular attention should be paid to the step base in terms of pressure loads, since the vicinity of the step base represents a zone of strong compression.

In an effort to highlight the points of interest, pressure ratio contour maps around the gap/step configuration are demonstrated in Fig. 6 (right column) parameterized by the frontal face $h^{*}$. On examining Fig. 6 (right column), two interesting features are noticed. One of them is related to the peak value for pressure ratio close to the shoulder at the frontal face. It is clearly seen that this peak value increases by increasing the frontal-face height $h^{*}$. Indeed, the reason for this is that the location of these peak values is related to the flow reattachment point on the frontal-face surface. The other one is the pressure 
rise inside the gap due to the presence of the step. According to Paolicchi [38], the pressure ratio $p / p_{\infty}$ in the gap, for the $L / H=1 / 4$ case, basically changes from 29 (at the top) to 12 (at the bottom). Nevertheless, for the gap/step configuration, Fig. 6 (right column), the pressure ratios are about 130, 230, and 280 for frontal face $h^{*}$ of $3.23,6.46$, and 9.69, respectively. In this scenario, it is firmly established the combination of a step with a gap increases the pressure ratio inside the gap one order of magnitude when compared to that for the gap only.

\subsection{Temperature Field}

In a diatomic or polyatomic gas in complete thermodynamic equilibrium, the translational temperature is equal to the temperature related to the internal modes, i.e., rotational, vibrational, or electronic temperatures, and it is identified as thermodynamic temperature. When the equilibrium is disturbed, relaxation processes arise in the system that try to return it to the state of the total statistical equilibrium. In diatomic or polyatomic gas, there are the processes which result in the establishment of equilibrium with respect to individual molecular degree of freedom, such as translational, rotational, vibrational, or electronic. Conversely, in a thermodynamic nonequilibrium gas, an overall kinetic temperature is defined as the weighted mean of the translational and internal temperatures [18] as follows:

$$
T_{O}=\frac{\zeta_{T} T_{T}+\zeta_{R} T_{R}+\zeta_{V} T_{V}}{\zeta_{T}+\zeta_{R}+\zeta_{V}}
$$

where $\zeta$ is the molecular degree of freedom and subscripts $T, R$, and $V$ stand for translation, rotation, and vibration, respectively.

In order to have a clear qualitative picture of the temperature field, it is instructive to examine separately translational, rotational, and vibrational kinetic temperature profiles instead of the overall kinetic temperature $T_{O}$, as defined by Eq. (1). In this fashion, gap/step configuration effects on translational, rotational, and vibrational kinetic temperatures are illustrated in Figs. 7, 8, and 9, respectively, for three sections along surface S1. In this group of plots, temperature ratio stands for translation temperature $T_{T}$, rotational temperature $T_{R}$, and vibrational temperature $T_{V}$, normalized by the free-stream temperature $T_{\infty}$. In additon, $X, Y$, and $h^{*}$ are the dimensionless variables as defined previously in the density ratio profiles. Again, for comparison purpose, temperature profiles for the flat-plate case, for the gap [15], and for the forward-facing step [16] are also illustrated in the same group of plots. Furthermore, for forward-facing steps, the profiles are only shown for $h^{*}$ of 3.23 and 9.69. Again, results for the $h^{*}=6.46$ case are intermediate and they are not shown. 


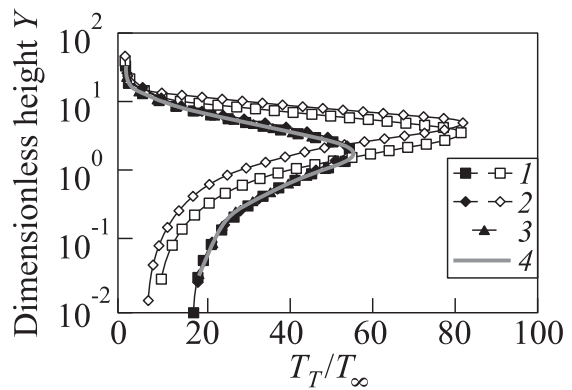

(a)

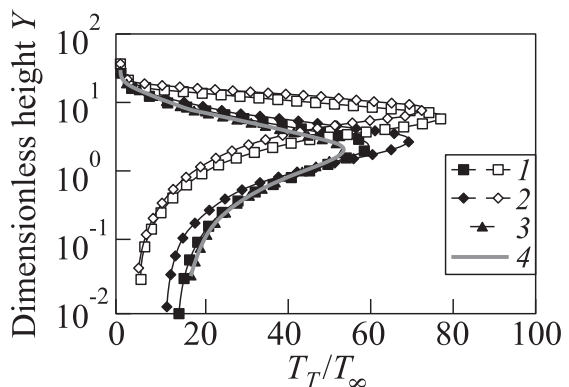

(b)

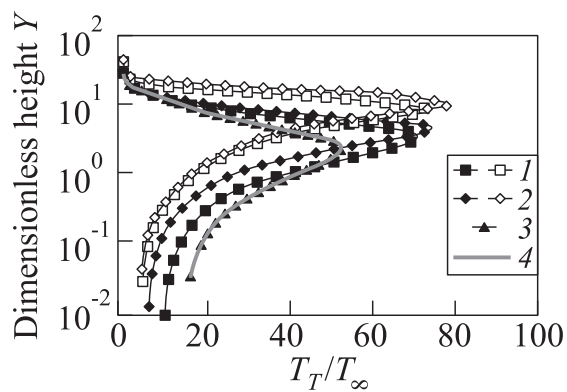

(c)

Figure 7 Translational temperature ratio $\left(T_{T} / T_{\infty}\right)$ profiles for three sections on surface $\mathrm{S} 1$ of the gap/step configurations (1 - gap/step; 2 - forward-facing; and 3 - gap) defined by section $X$ of $39(a)$, $44(b)$, and $48(c)$. Filled symbols refer to $h^{*}=3.23$; empty symbols refer to $h^{*}=9.69 ; 4$ - flat-plate case

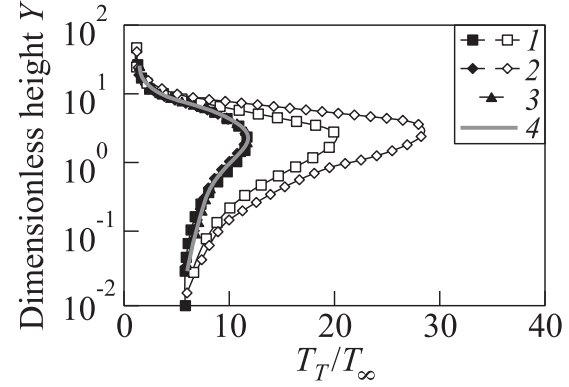

(a)

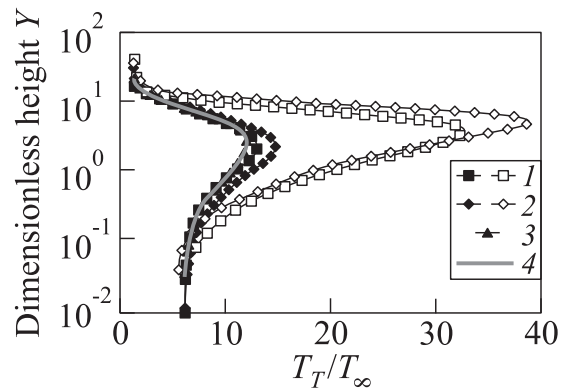

(b)

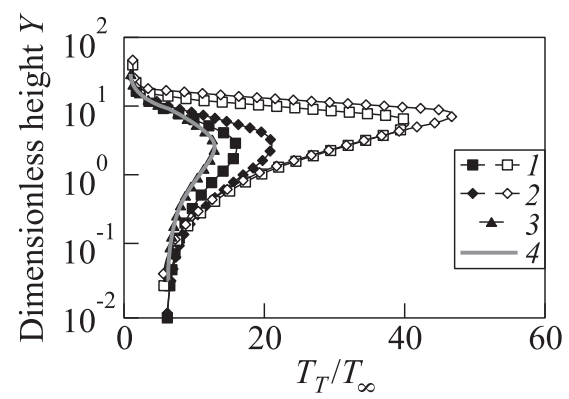

(c)

Figure 8 Rotational temperature ratio $\left(T_{R} / T_{\infty}\right)$ profiles for three sections on surface $\mathrm{S} 1$ of the gap/step configurations (1-gap/step; 2 - forward-facing; and 3 - gap) defined by section $X$ of $39(a)$, $44(b)$, and $48(c)$. Filled symbols refer to $h^{*}=3.23$; empty symbols refer to $h^{*}=9.69 ; 4$ - flat-plate case 


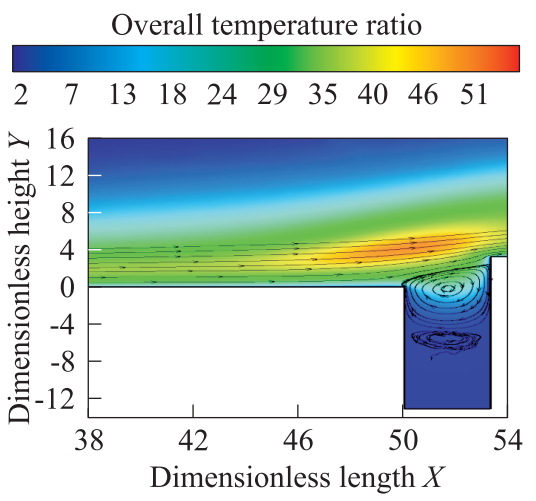

(a)

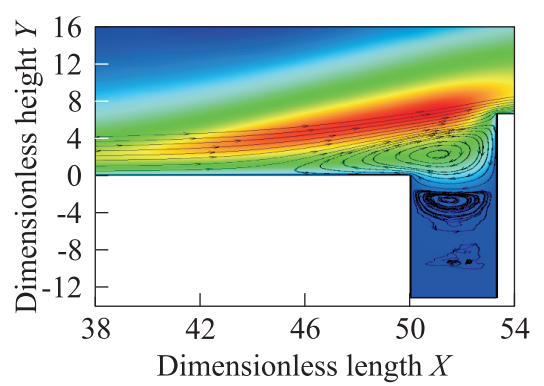

(b)

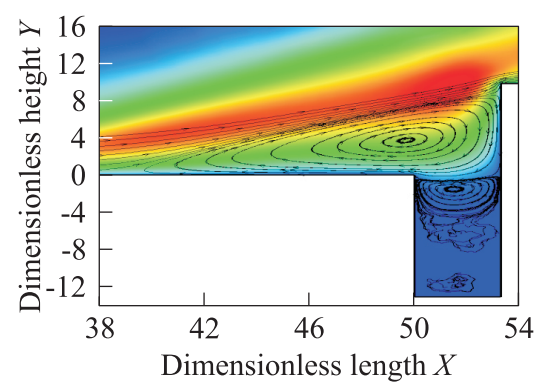

(c)

Figure 9 Vibrational temperature ratio $\left(T_{V} / T_{\infty}\right)$ profiles for three sections on surface S1 of the gap/step configurations (1 - gap/step; 2 - forward-facing; and 3 - gap) defined by section $X$ of $39(a)$, $44(b)$, and $48(c)$. Filled symbols refer to $h^{*}=3.23$; empty symbols refer to $h^{*}=9.69 ; 4$ - flat-plate case

Figure 10 Overall temperature ratio $\left(T_{O} / T_{\infty}\right)$ contour maps along with streamline traces at the vicinity of the gap/step configuration for step height $h^{*}$ of $3.23(a)$, $6.46(b)$, and $9.69(c) ; L / H=1 / 4$ 
On examining this group of plots, it is quite apparent that thermodynamic nonequilibrium occurs throughout the shock layer as shown by the lack of equilibrium of the translational and internal kinetic temperatures. The overall kinetic temperature $T_{O}$ is equivalent to the thermodynamic temperature only in thermal equilibrium conditions. As a matter of fact, it should be noticed that the ideal gas equation of state does not apply to this temperature in a nonequilibrium situation.

Referring to Figs. 7-9, it is clearly noted that in the undisturbed free-stream far from surface S1, $Y \rightarrow \infty$, the translational and internal kinetic temperatures have the same value and are equal to the thermodynamic temperature. Approaching surface S1, in the range $2<Y<10$, the translational kinetic temperature rises well above the rotational and vibrational temperatures and reaches the maximum value that relies on the geometry, i.e., flat plate, gap, forward-facing step, or gap/step configuration. In addition, this maximum value depends on section $X$. Since a large number of collisions is needed to excite vibrational modes of the molecules, from the ground state to the upper state, the vibrational temperature is seen to increase much more slowly than rotational temperature. Still further toward surface $\mathrm{S} 1, Y \approx 0$, the translational, rotational, and vibrational temperatures decrease and reach the values that depend on the section $X$. For section $X=39$, kinetic temperatures reach the values on the wall that are above the wall temperature $T_{w}\left(\approx 4 T_{\infty}\right)$, resulting in a temperature jump as defined in the continuum formulation. For section $X=44$, the difference between translational temperature and internal temperatures for the $h^{*}=9.69$ case indicates that the thermodynamic equilibrium is achieved close to surface S1. Finally, for section $X=48$, the kinetic temperatures basically reach the wall temperature $T_{w}$ and the thermodynamic equilibrium is achieved for the cases defined by $h^{*}$ of 3.23 and 9.69 .

In what follows, it proves convenient to present contour maps for temperature at the vicinity of the gap/step configuration. In doing so, Fig. 10 displays the overall kinetic temperature ratio $T_{O} / T_{\infty}$ parameterized by the step height $h^{*}$. Of particular interest in this group of plots is the impact of the step frontal-face height on the temperature behavior ahead of the step. It is clearly seen that temperature in this region increases with increasing the step frontal face for the conditions investigated.

\section{CONCLUDING REMARKS}

Computations of a rarefied hypersonic flow on gap/step configurations have been performed by using the DSMC method. The calculations provided information concerning behavior of the primary flow properties around a gap/step configuration located on a flat plate. Effects of the step frontal-face height on ve- 
locity, density, pressure, and temperature were investigated for a representative range of parameters. The gap length-to-depth $L / H$ ratio was set at $1 / 4$, and the step frontal-face height $h$ ranged from 3 to $9 \mathrm{~mm}$, which corresponded to Knudsen numbers in the transitional flow regime. In addition, results for the gap/step configuration were compared to those obtained for a flat plate, a gap, and a forward-facing step. It was found that separation region ahead of the gap/step configuration is a function of the step frontal-face height, i.e., it increases with increasing the step frontal-face height. The analysis showed that the size of the recirculation region for the gap/step configuration is similar to that for the forward-facing step with the same frontal-face height. It was also found that density and pressure for the gap/step configuration dramatically increased inside the gap as compared to those observed for the gap configuration, i. e., a gap without a step.

\section{ACKNOWLEDGMENTS}

The second author would like to thank the financial support provided by Capes (Coordenação de Aperfeiçoamento de Pessoal de Nível Superior) under Grant No. 2848/13-9, in order to attend the 5th European Conference for Aeronautics and Space Sciences.

\section{REFERENCES}

1. Bertran, M.H., and M. M. Wiggs. 1963. Effect of surface distortions on the heat transfer to a wing at hypersonic speeds. AIAA J. 1(6):1313-1319.

2. Rogers, E. W.E., and C. J. Berry. 1965. Research at the NPL on the influence at supersonic speeds and low Reynolds numbers of thick laminar boundary layers. Advances in applied mechanics: Rarefied gas dynamics. New York, NY, USA: Academic Press. I(Suppl. 3):574-591.

3. Weinstein, I., D. E. Avery, and A. J. Chapman. 1975. Aerodynamic heating to gaps and surfaces of simulated reusable-surface-insulation tile arrays in turbulent flow at Mach 6.6. NASA TM X-3225.

4. Wieting, A. R. 1978. Experimental investigation of heat-transfer distributions in deep cavities in hypersonic separated flow. NASA TN D-5908.

5. Avery, D. E. 1978. Aerodynamic heating in gaps of thermal protection system tile arrays in laminar and turbulent boundary layers. NASA TP 1187.

6. Bertin, J. J., and A. Keisner. 1978. Effect of step and/or gap tile misalignment on shuttle transition. NASA-CR-151833.

7. Scott, C. D., and R. J. Maraia. 1979. Gap heating with pressure gradients. AIAA Paper No. 79-1043. 
8. Smith, D. M., D. N. Petley, C. L. W. Edwards, and A. B. Patten. 1983. An investigation of gap heating due to stepped tiles in zero pressure gradient regions of the shuttle orbiter thermal protection system. AIAA Paper No. 83-0120.

9. Avery, D. E., P. A. Kerr, and A. R. Wieting. 1983. Experimental aerodynamic heating to simulated shuttle tiles. NASA TM-84654.

10. Petley, D. H., D. M. Smith, C. L. W. Edwards, A. B. Carlson, and H. H. Hamilton II. 1984. Surface step induced gap heating in the shuttle thermal protection system. J. Spacecraft Rockets 11:156-161.

11. Avery, D.E. 1985. Experimental aerodynamic heating to simulated shuttle tiles in laminar and turbulent boundary layers with variable flow angles at a nominal Mach number of 7. NASA TP-2307.

12. Hunt, L. R., and K.K. Notestine. 1990. Aerodynamic pressure and heating-rate distributions in tile gaps around chine regions with pressure gradients at a Mach number of 6.6. NASA TP-2988.

13. Charbonnier, J., and H. Boerrigter. 1993. Contribution to the study of gap induced boundary layer transition in hypersonic flow. AIAA Paper No. 93-5111.

14. Hinderks, M., R. Radespiel, and A. Gülhan. 2004. Simulation of hypersonic gap flow with consideration of fluid structure interaction. AIAA Paper No. 2004-2238.

15. Paolicchi, L. T. L. C., and W.F. N. Santos. 2013. Length-to-depth ratio effects on aerodynamic surface quantities of a rarefied hypersonic gap flow. AIAA Paper No. 2013-2789.

16. Leite, P.H. M., and W.F. N. Santos. 2011. Computational analysis of rarefied hypersonic flow over a forward-facing step. AIAA Paper No. 2011-3310.

17. Leite, P. H. M., and W.F. N. Santos. 2013. Computational analysis of low-density hypersonic flow over backward-facing steps. AIAA Paper No. 2013-2788.

18. Bird, G. A. 1994. Molecular gas dynamics and the direct simulation of gas flows. Oxford University Press. 458 p.

19. Harvey, J. K. 1986. Direct simulation Monte Carlo method and comparison with experiment. Rarefied gas dynamics: Thermophysical aspect of re-entry flows. Eds. N. Moss and C. D. Scott. Progress in astronautics and aeronautics ser. Washington, D.C., USA: AIAA. 103:25-43.

20. Moss, J. N., J. M. Price, V. K. Dogra, and D. B. Hash. 1995. Comparison of DSMC and experimental results for hypersonic external flows. AIAA Paper No. 95-2028.

21. Harvey, J. K., and M. A. Gallis. 2000. Review of code validation studies in highspeed low-density flows. J. Spacecraft Rockets 37:8-20.

22. Bird, G. A. 1981. Monte-Carlo simulation in an engineering context. Rarefied gas dynamics. Ed. S. S. Fisher. Progress in astronautics and aeronautics ser. New York, NY, USA: AIAA. 74:239-255.

23. Bird, G. A. 1989. Perception of numerical method in rarefied gasdynamics. Rarefied gas dynamics: Theoretical and computational techniques. Eds. D. P. Weaver, D. H. Campbell, and E. P. Muntz. Progress in astronautics and aeronautics ser. New York, NY, USA: AIAA. 118:374-395.

24. Borgnakke, C. and P. S. Larsen. 1975. Statistical collision model for Monte-Carlo simulation of polyatomic gas mixture. J. Comput. Phys. 18:405-420. 
25. Boyd, I. D. 1998. Analysis of rotational nonequilibrium in standing shock waves of nitrogen. AIAA J. 28:1997-1999.

26. Bird, G. A. 2009. A comparison of collision energy-based and temperature-based procedures in DSMC. 26th Symposium (International) on Rarefied Gas Dynamics. American Institute of Physics. 245-250.

27. Alexander, F. J., A. L. Garcia, and B. J. Alder. 1998. Cell size dependence of transport coefficient in stochastic particle algorithms. Phys. Fluids 10:1540-1542.

28. Alexander, F. J., A. L. Garcia, and B. J. Alder. 2000. Erratum: Cell size dependence of transport coefficient in stochastic particle algorithms. Phys. Fluids 12:731-731.

29. Garcia, A. L., and W. Wagner. 2000. Time step truncation error in direct simulation Monte Carlo. Phys. Fluids 12:2621-2633.

30. Hadjiconstantinou, N. G. 2000. Analysis of discretization in the direct simulation Monte Carlo. Phys. Fluids 12:2634-2638.

31. Chen, G., and I. D. Boyd. 1996. Statistical error analysis for the direct simulation Monte Carlo technique. J. Comput. Phys. 33:434-448.

32. Chew, Y.T., C. Shu, and X.H. Mao. 2005. Particle number per cell and scaling factor effect on accuracy of DSMC simulation of micro flow. Int. J. Numer. Method. Heat Fluid Flow 15:827-841.

33. Everhart, J. L., S. J. Alter, N. R. Merski, W. A. Wood, and R. K. Prabhu. 2006. Pressure gradient effects on hypersonic cavity flow heating. AIAA Paper No. 20060185.

34. Camussi, R., M. Felli, F. Pereira, G. Aloisio, and A. DiMarco. 2008. Statistical properties of wall pressure fluctuations over a forward-facing step. Phys. Fluids 20:075113.

35. Kim, H. D., and T. Setoguchi. 2007. Shock-induced boundary layer separation. 8th Symposium (International) on Experimental and Computational Aerothermodynamics of Internal Flows Proceedings. Lyon, France.

36. Deepak, N.R., S.L. Gai, and A. J. Neely. 2010. A computational study of high enthalpy flow over a rearward facing step. AIAA Paper No. 2010-0444.

37. Leite, P. H. M. 2009. Direct simulation of the step influence on a reentry vehicle surface. INPE (National Institute for Space Research), Combustion and Propulsion Laboratory. M.S. Thesis. [In Portuguese.]

38. Paolicchi, L. T. L. C. 2010. Computational analysis of gap effects on the surface of reentry space vehicles. INPE (National Institute for Space Research), Combustion and Propulsion Labaratory. M.S. Thesis. 